INDIVIDUOS Y ESTADIOS: UNA PROPUESTA FORMAL DESDE LA INFORMACIÓN ASPECTUAL DE LOS PREDICADOS

\title{
INDIVIDUOS Y ESTADIOS: UNA PROPUESTA FORMAL DESDE LA INFORMACIÓN ASPECTUAL DE LOS PREDICADOS ${ }^{1}$
}

\author{
Mario Casado Mancebo \\ Universidad Complutense de Madrid (España) \\ mcasad04@ucm.es
}

Recibido: 24/02/2019 - Aprobado: 12/06/2019

DOI: doi.org/10.17533/udea.lyl.n76a03

\begin{abstract}
Resumen: En este trabajo se ofrece una revisión teórica crítica de las categorías de individuo y estadio y de los diferentes enfoques que se les ha dado a ambas categorías desde los diferentes ámbitos de la gramática. Luego, se propone un análisis formal de los dos tipos de predicaciones desde la perspectiva de la información aspectual de los predicados desde la información de la que constan al salir del léxico, para concluir que la «coacción léxica» es una coacción aspectual que insertaría un Sintagma Aspecto con una variable espaciotemporal, interfiriéndose en la estructura funcional de las estructuras referenciales de la predicación.
\end{abstract}

Palabras clave: predicados de individuo; predicados de estadio; interfaz léxico-sintaxis; predicación; estructura aspectual.

\section{INDIVIDUALS AND STAGES: A FORMAL APPROACH FROM THE PREDICATES ASPECTUAL INFORMATION}

\begin{abstract}
One of the most controversial issues on studies about predication theory is the division between Individual Level Predicates and Stage Level Predicates as for whether the origin of the distinction is lexical, syntactic or semantic. This paper shows, at first, a theoretical, critical review of the two categories Individuals and Stages - and the various approaches given to them from the different fields of grammar. In second place, the article proposes a formal analysis of the two types of predicates from an approach focused on the aspectual structure, starting from the features that the items hold when they are drawn from the lexicon.
\end{abstract} Key words: individual level predicates; stage level predicates; lexis-syntax interface; predication theory; aspectual structure.

1. Un agradecimiento a la Dra. María Jesús Fernández Leborans y al Dr. Luis García Fernández por su dirección, revisión, consejos y ayuda inestimables durante la realización de este trabajo.

LINGÜÍSTICA Y LITERATURA

72

ISSN 0120-5587

E-ISSN 2422-3174

N. ${ }^{o} 76,2019,72-96$ 


\section{Introducción}

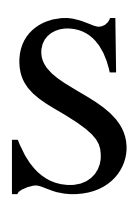

i hay un tema candente relativo a la predicación, es el referido a la oposición entre predicados de individuo y predicados de estadio. Esta distinción, que surge como una distinción semántica entre los predicados que denotan propiedades y los que denotan estados, ha rebasado los límites de la semántica y ha alcanzado los debates sobre el léxico y la sintaxis. En unos casos, se ha buscado la presencia de algún rasgo en el léxico que determine el tipo de predicación que se proyectará en la sintaxis. En esta línea, se puede señalar a Davidson (1967), quien propuso el argumento eventivo, como primera aproximación a la diferencia de predicados. Esta propuesta ha sido muy debatida, hasta el punto de que algunos autores propondrían que tal argumento está presente en todos los predicados y que se distribuye entre los constituyentes de la predicación. Para otros, el argumento eventivo es, precisamente, la forma de distinguir entre predicados de individuo y de estadio: los primeros estáticos [- dinámicos] y los segundos dinámicos [+ dinámicos]. Este enfoque tiene la ventaja de integrar los dos tipos de predicados en la teoría vendleriana de la estructura eventiva de los verbos. Una de las limitaciones de esta teoría (Vendler, 1967) es que con su división entre estados y eventos a través del rasgo [ \pm dinámico] no podía dar cuenta de la división que notó Milsark (1974) entre predicados de individuo y de estadio.

Con las ideas de principios y parámetros sobre la estructura subatómica del léxico (Pustejovsky, 1995), surge una nueva fuente de discusión: si se considera que la distinción emana de un argumento eventivo, se está localizando el tipo de predicado en la estructura argumental del léxico. Puesto que en esta capa de información léxica se codifica un tipo de información más configuracional que de significado, no es una hipótesis satisfactoria. Si se tiene en cuenta el tipo de información que la distinción entre individuo y estadio proporciona, que, como se veía anteriormente, está relacionada con la estructura eventiva de los predicados, se puede proponer que se está operando en la capa de la estructura aspectual del léxico y que en ella existe algún tipo de información que se deriva en estados y propiedades. 
Por otro lado, la presencia de algún tipo de información en el léxico como fuente de la oposición entre predicados ha llevado a considerar si existen casos en los que alguna predicación se defina como individuo o estadio como producto de la sintaxis y no simplemente de la información léxica que se proyecta. En el seno de esta propuesta surge la llamada coacción aspectual (Pustejovsky, 1995), que permite la interacción de rasgos y capas en el interior del léxico de los hablantes. Es una propuesta profundamente semántica que, por lo tanto, no tiene en cuenta la sintaxis. Este hecho ha llevado a redefinir el concepto de coacción aspectual desde distintas perspectivas más allá del léxico.

En el apartado 2, se repasará en profundidad las formas de concebir la predicación como fenómeno gramatical, de forma que se pueda establecer una frontera nítida entre el léxico y la sintaxis. En estos términos, se podrá entender correctamente la noción de coacción, su origen y sus consecuencias. El apartado 3 se centrará concretamente en la distinción individuo-estadio como parte de la predicación gramatical. En primer lugar, se expondrán los principales enfoques que se han dado a las dos formas de predicación, sus ventajas y sus inconvenientes para, finalmente, buscar el que dé cuenta de la oposición PI-PE de la forma más completa. Seguidamente, se buscará el origen de la distinción: ¿está en el léxico? ¿Está en la sintaxis? En último lugar, se propondrá una hipótesis formal que dé cuenta de los diferentes aspectos concluidos a través del trabajo. En la última sección, la número 4, se retomará la discusión a modo de cierre para recopilar todas las conclusiones que se habrán extraído a lo largo del trabajo.

\section{La predicación}

Cuando se habla de predicación, la discusión se suele abordar directamente desde los conceptos de predicado de individuo (PI) y predicado de estadio (PE) (Milsark, 1974), que si bien, efectivamente, suponen un amplio campo dentro de los estudios sobre predicación, no se puede olvidar la propia noción de predicado. Aunque parece un término transparente por su actual aceptación y uso generalizado, en muchos análisis algunas nociones semánticas y sintácticas relacionadas con él conducen a un análisis erróneo de los predicados de individuo y los de estadio. Por ello, antes de sumergirse en la distinción entre 
PI y PE, conviene aclarar una serie de conceptos fundamentales relacionados con la predicación.

\subsection{La predicación semántica}

El concepto de predicación tiene una carga muy grande de nociones que parten de la semántica; es decir, de clasificaciones basadas en el significado de las unidades lingüísticas. En este trabajo, para hacer referencia a los aspectos semánticos de la predicación, se adoptará una posición representacionalista, dado que es la que más ventajas ofrece a la hora de analizar los predicados, las expresiones referenciales y su utilización en el lenguaje. En este enfoque, se entiende que el significado de las expresiones está relacionado con la imagen mental que cada individuo asigna a un concepto. Se puede ir más allá de la mera referencia de las palabras y distinguir entre la intensión o sentido de los predicados, la representación mental de los rasgos necesarios —en el sentido lógico- de esa entidad, que son los que habilitan la posibilidad de evaluar las condiciones de verdad de la expresión referencial; y la extensión o referencia de los predicados, el conjunto de entidades en las que se puede evaluar de forma verdadera la intensión de una expresión lingüística.

De esta forma, se da por sentado que la expresión predicativa o predicado corresponde a una intensión, es decir, a un evaluador de conjuntos. Una expresión que, por sí misma, no es capaz de referir a algo determinado. Los nombres comunes, per se, son predicados ${ }^{2}$. Una expresión como mesa no es capaz de identificar ningún elemento de forma unívoca. Las expresiones referenciales o singulares, por su lado, en una teoría de este corte son aquellas capaces de identificar unívocamente la imagen mental de un individuo del mundo real.

Resulta evidente que, si las expresiones quedasen divididas en los dos compartimentos estancos que se acabaron de definir (predicados y expresiones referenciales), sería imposible expresar más ideas que los meros conceptos que las palabras contienen. Sería imposible construir proposiciones con significados complejos, ya que, teniendo en cuenta

2. Los nombres propios se comportan de modo diferente, puesto que, aunque tradicionalmente se los agrupa bajo la etiqueta de sustantivo o nombre por haber sido entendidos como elementos que designan entidades, tienen propiedades y muestran comportamientos diferentes de los nombres comunes.

LINGÜÍSTICA Y LITERATURA

ISSN 0120-5587

E-ISSN 2422-3174

N. ${ }^{\circ} 76,2019,72-96$ 
que hasta los propios nombres son categorías predicativas, la capacidad de referencia quedaría restringida a una mínima lista de unidades. Sin embargo, la capacidad referencial de las expresiones va más allá de la que intrínsecamente contienen las unidades del léxico. Si bien hasta ahora se está situado en el primer nivel del sistema del lenguaje (el léxico), el componente sintáctico permite realizar una serie de operaciones de ensamble. Cuando se habla del ensamble, en general, es para hablar de la computación sintáctica; sin embargo, bien se puede afirmar que la operación de ensamble está presente incluso en los niveles más básicos de nuestra capacidad ${ }^{3}$.

La manifestación más clara del ensamble conceptual es la selección temática en el léxico. Para adquirir complejidad y, de esa forma, ir acercándose a la capacidad referencial que legitime su computabilidad sintáctica, los predicados contienen en el léxico una serie de criterios de selección temática que rigen su saturación de significado; esto es, para ir adquiriendo la complejidad conceptual necesaria que conforme una expresión capaz de referir, los predicados, en su estructura temática del léxico, requieren una serie de elementos que no pertenecen puramente a la esfera lingüística, sino a primitivos relacionados con nuestra concepción de los eventos: agentes, temas, benefactivos, etc.

\subsection{La predicación sintáctica}

Como se desprende de lo dicho, no se va a considerar, como en la gramática tradicional, que la predicación esté reducida al ámbito del sintagma verbal. Se asumirá la idea de Bosque y Gutiérrez-Rexach (2009): «Será una estructura predicativa cualquier relación de saturación entre una función proposicional y su argumento» (p. 260). Los autores entienden los predicados como funciones proposicionales en el sentido matemático $f(x)$ : un elemento $f$ que opera sobre otro $x$. El producto del segundo depende del valor del primero. Esta «funcionalidad» del ensamble predicativo es lo que da cuenta de por qué, cuando se saturan predicados de forma recursiva, no se obtiene una suma. Se puede obtener un producto: la

3. Eguren Gutiérrez y Fernández Soriano (2004, pp. 56-57) propusieron, precisamente, como un posible origen del lenguaje, esa habilidad de ensamble. Pero no un ensamble lingüístico, sino uno conceptual.

LINGÜÍSTICA Y LITERATURA

ISSN 0120-5587

E-ISSN 2422-3174

N. ${ }^{\circ} 76,2019,72-96$ 
suma de sus partes y algo más. Es lo que se conoce como principio de composicionalidad, enunciado por Frege (1952). Ese «algo más» es el ensamble, proporcionado por la sintaxis.

Los sustantivos, como se mencionó en la sección inicial, también son predicados, por lo que, para obtener un elemento computable, habría que ensamblarlos con un argumento. El hecho de que los sintagmas nominales escuetos no sirvan en todos los contextos como constituyente sintáctico utilitario fue lo que llevó en el marco de principios y parámetros a proponer la categoría del sintagma determinante, una estructura de tipo nominal, pero con capacidad referencial: una expresión singular (ver sección 2.1).

La función abstracta o primitiva que se aplica tanto en la selección de argumentos como en la saturación por referencia de los nombres es el ensamble. Aunque, en las primeras formalizaciones de la gramática generativa, las operaciones sintácticas son dos: ensamble (del inglés merge) y movimiento (del inglés move) (Chomsky, 1995); al final, se ha hecho evidente que es la misma operación de fusión (Bosque y Gutiérrez-Rexach, 2009, p. 222) o simplemente ensamble (Brucart \& Hernanz, 2015, p. 59). Chomsky (2001) habló ya de la operación del movimiento como ensamble interno. Al fin y al cabo, el movimiento no es más que una operación de copia de rasgos por requisito de ensamble en una posición más alta. Este requisito de los rasgos, de forma abstracta, es también una forma de relaciones de saturación predicativa. Si bien es cierto que no es la clásica relación predicativa (la de corte léxico), también es cierto que, a lo largo de esta sección, se ha visto cómo las exigencias de los predicados no acaban, ni mucho menos, en el léxico. De igual forma que un sintagma nominal escueto requiere un elemento funcional como es la determinación y esto se asume como parte de la red predicativa de los nombres, según se va subiendo en la estructura hacia la zona del tiempo, los requisitos predicativos son más complejos. Sin embargo, el que los mecanismos predicativos no estén tan próximos al léxico en esa zona no tiene que ser un motivo para considerarlo un mecanismo distinto. Ya se ha comprobado que el propio Chomsky reconoció que la motivación del movimiento no es más que otro ensamble.

Entonces, se debe concluir que, una vez que se encuentren categorías gramaticales que se combinan siguiendo las restricciones de selección léxica, como se veía en el apartado 2.1., se estará ante un tipo de predicación regida por las sintaxis, ya que el mecanismo que opera es el ensamble. De esta forma, se debe tener claro que, desde una perspectiva 
lexicista, todas las relaciones de ensamble desde el que se extrae un ítem del léxico hasta que se culmina la última fase de derivación gramatical son predicaciones sintácticas. Sobre todas ellas operan reglas de computación (el componente sintáctico). Es importante tener esto en cuenta, esencialmente, por dos motivos: en primer lugar, la revisión de la predicación de individuo y de estadio que se va a realizar a continuación adoptará una perspectiva formal, que se centrará en una predicación de corte especialmente sintáctico. Por lo tanto, será necesario entender claramente dónde se establece la línea entre la predicación semántica, que viene desde el léxico, y la predicación sintáctica, que está determinada por lo que sucede una vez que las piezas salen del léxico; en segundo lugar, porque la propuesta formal que se formulará como una posible manera de recoger la diferencia entre PI y PE estará determinada por las operaciones que tiene lugar en la sintaxis, es decir, la sintaxis determinará la semántica y no al contrario, como sucedería desde el punto de vista recogido en el apartado preliminar.

\section{La oposición entre el predicado de individuo y el predicado de estadio}

\subsection{Marco teórico}

La distinción de predicados en la categorización individuo-estadio parte de la tesis doctoral de Milsark (1974), quien sugiere distinguir las relaciones de predicación en aquellas que expresan propiedades (en inglés properties) y aquellas que expresan estados (en inglés conditions). El primer tipo define las relaciones de tipo inherente al sujeto de la predicación y, por tanto, se asumen como permanentes e inalterables en el tiempo. El segundo tipo define las relaciones de tipo no inherentes, es decir, aquellas que no se consideran cualidades esenciales del sujeto predicativo. Al contrario que las anteriores, por no ser inherentes al sujeto, se interpretan como transitorias.

De esta clasificación inicial, parte la concepción tradicional de que los PI son permanentes y los PE son transitorios y, para el español, la división de ser y estar basada en estas nociones: ser para estados permanentes y estar para los transitorios. Por ejemplo: 
1) María es orgullosa.

2) María está orgullosa.

Los casos 0 y 0 podrían constituir un ejemplo de la distinción permanente-transitorio de Milsark. Mientras que 0 denota una propiedad inherente o definitoria de María, 0 denota un estado, un momento determinado en el que María pasa por una cualidad transitoria. Así, 0 sería un PI y 0, un PE. Como se verá a continuación, ni la dicotomía PI-PE tiene su fundamento en la temporalidad, ni ser y estar son un resultado de ella.

Carlson (1977) notó el problema de tomar la permanencia temporal del valor de la predicación como base de la distinción PI-PE. Como recogió Fábregas (2012), un buen ejemplo es la predicación sobre los adjetivos vivo y muerto.

(1) Juan está $\{$ vivo/muerto $\}$

Los predicados del caso 0 evidentemente son persistentes en el tiempo. No son categorizables como transitorios. Esto debería hacerlos propiedades según la clasificación de Milsark. Sin embargo, como bien se sabe, son estados — siempre según el razonamiento de Milsark-, ya que no es una propiedad inherente a Juan el estar vivo o muerto. Si Juan perdiese la cualidad de «estar vivo» no dejaría de ser reconocido como el individuo Juan; y, por supuesto, que, inexorablemente, llegará un momento en el que Juan cambie de estado, para pasar a estar muerto. En ese momento, Juan seguirá siendo Juan, por lo que no parece que vivo/muerto formen parte del repertorio de rasgos identificativos del individuo.

Como solución, Carlson (1977) planteó reconfigurar la oposición PI-PE. Para Milsark (1974), PI se oponía directamente a PE. Simplemente, suponían nociones diferentes (paralelas, se podría decir). Carlson nota que en realidad no se trata de una oposición de nociones isovalentes, sino que una de las dos es primitiva. Y, dado que los PI establecen una relación que, en términos de hoy, se podría denominar intensional, en el sentido de que atribuye a una expresión unas propiedades que se interpretan como definitorias del individuo que sea, es esta categoría la que el autor propone como básica. Los PI constituyen, de esta forma, una relación predicativa directa. Si cuando se habla de una predicación semántica del tipo que se veía en la sección 2.1, se utiliza la notación de (1) 
para expresar la relación de saturación entre el predicado (guapo) y la expresión referencial de la que se predica el adjetivo, en el caso de los PI, sería similar. Una predicación como Juan es guapo tendría una estructura semántica y sintáctica (ver apartado 2) de relaciones estructurales directas (2):

(1) Guapo(x)

(2) Juan es guapo

a. Guapo (Juan)

b. Juan guapo

Los PE, sin embargo, serían una derivación sintáctica de un PI. Para expresar el estadio, habría que formar una predicación de segundo nivel sobre un PI. Para incluir en la representación el factor temporal de la transitoriedad, Carlson aseguró que la PI se forma sobre una variable espacio-temporal. De forma intuitiva, sería como predicar un adjetivo sobre una situación $(t)$, no sobre un individuo y, después, esa relación predicativa de primer orden se aplica al individuo.

(3) Juan está guapo ( $\rightarrow$ «Juan es guapo en una franja espacio-temporal»)

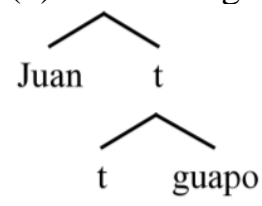

En conclusión, Carlson propuso que PI representa propiedades necesarias de los individuos y PE define, o más bien, predica estados o situaciones que se relacionan con individuos sin ser propiedades inherentes.

Kratzer (1989), por su parte, codificó argumentalmente la teoría siguiendo la línea de Carlson. La autora propuso que los PE incluyen la variable espacio-temporal (en este trabajo, $t$ ) como un elemento argumental de su configuración léxica, lo que les permite ser interpretados como eventualidades. Los PI, sin embargo, carecerían de esta variable, por lo que deben ser interpretados como estados planos y sin delimitación, puesto que sin la variable $t$, estarían desprovistos de coordenadas espacio-temporales que los acoten. Si a Kratzer le sirvió para distinguir dos tipos de estados, Hale y Keyser $(1987,1988)$ lo utilizaron para caracterizar estados (no dinámicos, sin $t$ ) frente a eventos. Arguyeron que 
solo los verbos con variable $t$ son capaces de denotar eventos dinámicos ${ }^{4}$, y aquellos que no cuenten con esa especificación solo pueden ser estáticos. Esto es de suma importancia para los predicados que se están tratando en el trabajo, ya que saca a los PE de los estados tradicionales (especificados con el rasgo [- dinámico]) y los clasifica como eventos.

Del análisis de Kratzer se desprende aún la influencia de la temporalidad de los estados en el análisis de estos. Aunque es partidaria de incluir la variable $t$, no deja de suponer que los PI constituyen predicaciones permanentes. Como señala Fábregas (2012), esto supone un problema para explicar ejemplos como Marta era rubia cuando era pequeña, que se comporta como un PI y, sin embargo, no es una predicación que coexista con el individuo («interval shorter than the life span of an individual», p. 10), puesto que de ella se infiere que actualmente ya no es rubia.

Fueron Higginbotham y Ramchand (1996) los que terminaron de perfilar la línea argumentativa sobre la predicación que se ha seguido hasta aquí. Estos autores consideraban, en línea de Carlson y Kratzer, que los PI son predicados definitorios (en inglés classificatory); es decir, definen subconjuntos de individuos. De nuevo, hablan sobre la capacidad intensional de los PI. La lectura permanente de este tipo de predicados no es más que una inferencia que realizan los hablantes. Al añadirse el predicado a lo que se conoce como valores intensionales del individuo («las propiedades lógicamente necesarias», vid. 2.1), se pasa a interpretar como una propiedad inherente y, por tanto, inmutable (Escandell Vidal y Leonetti, 2002). Sin embargo, nada en la gramática indica necesariamente que esas propiedades no puedan estar delimitadas. Como señalan los autores (ibidem), el adjetivo joven es definitorio-intensional y, por tanto, PI. Que Juan es joven sea gramatical se debe a que la permanencia de este predicado no está codificada en la gramática. Está en la pragmática a través de una inferencia generada por el rasgo de duratividad en el adjetivo joven. Aun cuando, objetivamente, el predicado joven no tiene una duración definida, puesto que es una propiedad que se pierde gradualmente en el

4. Nótese que para la distinción que propone Kratzer (1989) utilizo el término eventualidad, que no debe confundirse con los eventos — opuestos a los estados — de la clasificación de Vendler. Con eventualidad se hace referencia a un «proceso de tipo verbal» o a «la descripción de un evento». En el caso de Hale y Keyser $(1987,1988)$ se utiliza evento, porque ellos sí utilizan la variable $t$ para distinguir eventualidades dinámicas (eventos) y no dinámicas (estados).

LINGÜÍSTICA Y LITERATURA 
tiempo, se entiende que existe un periodo delimitado en el que las personas son jóvenes. De esta forma, se favorece la mencionada inferencia de duratividad.

Los PE, por su parte, añaden algo a las relaciones de PI: asocian propiedades a situaciones (Higginbotham \& Ramchand, 1996). De esta forma, como explicó Fábregas (2012), la predicación será válida, en términos veritativo-condicionales, tanto como lo sea $t$. Si ahora se recuerda la estructura predicativa de los PI, se notará que el predicado se asociaba directamente a su individuo. Esto provoca que la validez de esa predicación dure tanto como el individuo y provoque, como se mencionó en el párrafo anterior, una inferencia de propiedad permanente.

Como ha quedado claro, la variable $t$ o la inserción de «la situación» en el análisis es fundamental si se quiere captar la esencia de la verdadera diferencia entre PI y PE. Fue Kratzer, como se pudo apreciar, la que la propuso. Sin embargo, su idea de la eventividad se basó de las ideas de Davidson, quien propuso lo que se conoce como argumento eventivo (e), posteriormente denominado argumento davidsoniano (Davidson, 1967)

La propuesta de Davidson trató de resolver el problema de los complementos circunstanciales. Si bien hoy está claro el diferente estatuto de argumentos y adjuntos, en aquel momento aún constituía una incógnita el hecho de que a la estructura argumental de los verbos se pudieran añadir potestativamente otros constituyentes que, además, presentaban una gran flexibilidad en cuanto a la posición sintáctica. Como explicaron Bosque y Gutiérrez-Rexach (2009, p. 291), para una oración como (4), nadie aceptaría una formalización argumental semántica como la de (4)a, en la que se supondría que hay una relación de selección argumental entre cortar y las cinco de la tarde. En primer lugar, como se vio en la sección 2.2., se asume que la valencia máxima de un predicado es de tres argumentos; en segundo lugar, para interpretar el evento denotado por cortar no es necesario, en el sentido lógico-conceptual, ninguno de los constituyentes $z, u, v$ de la estructura (4)a. La formulación correcta es la que aparece en (4)b

(4) Juan cortó el pan con un cuchillo a las cinco de la tarde en la cocina.

a. $\operatorname{Cortar}\langle\mathrm{x}, \mathrm{y}, \mathrm{z}, \mathrm{u}, \mathrm{v}>$

b. $\operatorname{Cortar}<\mathrm{x}, \mathrm{y}>$ 
Como solución al problema de los complementos circunstanciales, Davidson planteó el argumento eventivo (o argumento $e$ ). Un argumento representante de la eventividad verbal, del que se harían depender los complementos circunstanciales. De esta forma, no formaban parte de una red argumental infinita del verbo. Pasaban a ser argumentos monovalentes que tomaban como sujeto de su cláusula predicativa el argumento $e$.

El argumento $e$ ha sido muy común en las hipótesis sobre la diferencia entre PI y PE. Desde Kratzer (1989) hasta Escandell Vidal y Leonetti (2002), pasando por Hale y Keyser (1987) y Higginbotham y Ramchand (1996), han defendido la importancia de esa temporalidad en los PE. Sin embargo, desde el comienzo, el argumento e ha sido también muy cuestionado. En sus orígenes se propuso como un argumento más de la estructura léxica de los predicados; sin embargo, esto supone un claro problema metodológico: si es un argumento de la red temática de los predicados, ¿dónde se proyecta al entrar en la sintaxis? Cabe recordar que según el principio de proyección (Chomsky, 1981, p. 29), la estructura proyectada en la sintaxis debe ser un reflejo de la estructura léxica de los predicados. Por lo tanto, que un argumento presente en el léxico desaparezca en la sintaxis, supone una grave contradicción a un principio tan sólido como este.

Si bien las ventajas del argumento eventivo a la hora de establecer una distinción tan primitiva como la de la dinamicidad permitieron obviar sus desventajas metodológicas, con la propuesta neodavidsoniana se puso en tela de juicio la verdadera necesidad de postular este argumento en el léxico. La corriente neodavidsoniana postuló que todos los predicados contenían un argumento e en el léxico (Higginbotham, 1985, 1989; Parsons, 1985, 1990; Schein, 1993). Evidentemente, si todos los argumentos contienen el argumento e, aparte de arrebatarle su valor teórico, se pierde la posibilidad de distinguir de forma explícita los predicados eventivos de los estativos. La polémica sobre qué predicados debe especificarse con el argumento $e$ ha desgastado mucho la credibilidad de la propuesta y ha contribuido a desdibujar, aún más, su significado. Por ello, en este trabajo, se hablará de la variable temporal o variable $t$. De esta forma, se dejará de lado la confusa denominación de argumento y, por consiguiente, cualquier polémica del objeto de estudio planteado.

La cuestión ahora es encontrar cómo se interpreta y a qué se asocia esa variable $t$. Horno Chéliz (2011) partió de una perspectiva semántica y asoció la presencia de la variable $t$ a la 
referencia, que se vincula, a su vez, con la presencia de un cuantificador existencial. En esta línea, se podría proponer que la variable $t$ se especifica en el sintagma tiempo (ST). Sin embargo, vale la pena recordar que, en realidad, todas las predicaciones con variable $t$ incluyen un sintagma tiempo en la zona de la flexión, por lo que sería extraño que unos ST tuvieran la variable y otras no, sobre todo, siendo una categoría funcional que, presumiblemente, debería ser mecánica.

Nótese el siguiente contraste:

(5)

a. María es alegre.

b. María está alegre.

(6)

a. Los leones son mamíferos.

b. Los leones están hambrientos.

En los dos casos de (5), hay un contraste basado en la distinción PI-PE. Si se piensa en la forma en la que están cuantificados en relación con lo que se ha comentado antes sobre la interpretación del predicado adjetival y su validez en relación a $t$, se notará que (5)a es una predicación homógenea: se presupone que siempre que María sea María, el predicado alegre se aplicará a María; en (5)b, sin embargo, se tiene una sección de evento. Puede considerarse a María como un evento $x$ en el tiempo. Si la predicación de (5)a se presuponía homogénea con respecto a María, la de (5)b que, sabiendo bien, incluye $t$, es un fragmento de $x$. Entonces, se tiene una predicación cuantificada. Sucede lo mismo con los casos de (6) y, si se observan con atención, la versión homogénea (6)a produce una lectura genérica y la cuantificada (6)b, una lectura existencial. Queda evidenciada, por tanto, la relación entre la cuantificación y la variable $t$.

Como se sabe, la cuantificación nominal tiene su propia categoría (SQ). No obstante, para la cuantificación de los eventos se propone que se manifieste a través del aspecto (sintagma aspecto, SAsp). Esto es así porque se ha observado que el caso acusativo es muchas veces responsable de la determinación aspectual de los predicados verbales como télicos (delimitados aspectualmente) o atélicos (no delimitados) (Husband, 2012). Si a eso se le añaden los asuntos sobre diátesis que también se han colocado en esta proyección, se apreciará que no es una idea descabellada atribuir la determinación, que como se vio, está 
íntimamente relacionada con la cuantificación, al SAsp. Así lo entiende Horno Chéliz (2011) aunque, si bien ella afirmó que todas las estructuras predicativas contienen un SAsp que puede estar especificado [ \pm dinámico], en esta ocasión se considerará que el SAsp implica la especificación [+dinámica] por defecto, y que su introducción en el cómputo sintáctico tiene consecuencias sobre la asignación de interpretación de individuo (homogéneo) o de estadio (cuantificado) en la forma lógica.

De esta forma, en esta propuesta se demostrará que la relación predicativa primitiva es la de PI y que, al añadirle o existir previamente una capa aspectual, esa predicación adquiere una interpretación de PE.

\section{2. ¿Dónde surge la distinción PI-PE?}

Habitualmente, como se mencionó al comienzo de la sección 3.1., la distinción entre PI y PE en español se ha hecho depender de la utilización de ser o estar en las predicaciones. Esta hipótesis crea una falsa presuposición de que estos verbos copulativos determinan si el producto de sus predicaciones se interpretará de una forma o de otra. Una presuposición de que estos verbos son primitivos a la distinción.

La anterior es una presuposición incorrecta, puesto que, en primer lugar, supone que el hablante selecciona del léxico uno u otro verbo para formar con ellos PI o PE. Teniendo en cuenta que estos verbos no imponen ningún tipo de restricción semántica a sus argumentos - si es que se puede denominar así a los constituyentes que coaparecen con un predicado que no impone requisitos de selección - no es apropiado atribuirles un papel tan esencial como el de determinar la interpretación de la oración. En segundo lugar, los datos reales no encajan en esta afirmación. Existen casos en los que estar denota PI y ser denota PE:

(7) PI con estar

a. Juan está loco.

b. Los mejillones están deliciosos.

(8) PE con ser

a. La reunión es arriba.

b. La fiesta es en el tercero. 
En (7), se puede apreciar cómo dos predicados que se incorporan a la intensión —es decir, se interpretan como propiedades definitorias - de sus individuos, se combinan con el verbo estar y no con ser, como habría de esperarse según la hipótesis tradicional mencionada en el párrafo anterior. En (8), de igual forma, unas propiedades que no parecen ser del repertorio intensional, puesto que las locaciones expresadas en el par parecen más bien circunstanciales - al mantenerse en la línea tradicional de este enfoque - que definitorias; y, sin embargo, se combinan con ser y no estar.

En tercer lugar, si la interpretación dependiera de ser o estar, deberían poder alternar libremente en las mismas estructuras para hacer posible una u otra lectura. Como se verá a continuación, esto no es posible. No parece que la distinción entre PI y PE esté predeterminada por la elección entre ser y estar. Más bien parece que ser y estar son la consecuencia del tipo de estructura que se haya construido en la sintaxis, ya que sobre la alternancia ser-estar operan toda una serie de restricciones que se explican teniendo en cuenta la categoría que se tome para construir la predicación. Parece lógico suponer que la asignación dependa, por un lado, de la categoría que tome la predicación primaria en la sintaxis. Los sintagmas determinantes (SD) y los sintagmas nominales (SN) no son combinables, con interpretación de atributo, con estar (Escandell Vidal y Leonetti, 2002). Los SD fuertes procuran lecturas identificativas (9) y los SD débiles y los SN, siempre se combinan con ser (10). Las categorías conflictivas, entonces, son los adjetivos (11) y los sintagmas preposicionales ${ }^{5}(12)$. Puesto que el objetivo de este trabajo no es un análisis de casos, no se tratarán de forma pormenorizada los distintos productos sintácticos y semánticos de las categorías mencionadas. Para un análisis exhaustivo, consúltese Escandell Vidal y Leonetti (2002)

(9)

a. Juan es el jefe del departamento.

b. Mi hermana es la chica más guapa de su clase. (10)

a. Jack Nicholson $\{$ es/*está $\}$ un actor de primera.

b. Juan es $\{$ es/*está $\}$ profesor.

5. La coacción aspectual de predicados no excluye a los sintagmas preposicionales, según Escandell Vidal y Leonetti (2002). Como se verá, la coacción sintáctica del aspecto siempre está determinada por las propiedades gramaticales del constituyente predicativo. 
(11)

a. Juan $\{$ es/está\} rubio.

b. Los noruegos son altos. (Fábregas, 2012)

c. Los noruegos están borrachos. (Fábregas, 2012)

(12)

a. María $\{$ es/*está $\}$ de Madrid.

b. María \{es/está\} de un inteligente.

c. María \{es/está\} de un tonto.

d. Mi casa $\{$ es/está $\}$ a la derecha.

e. Este sobre $\{$ es/?está $\}$ para ti.

En primer lugar, en (11) se observa que los adjetivos permiten con pocas restricciones la presencia de ser o de estar. Notablemente, proporcionan una interpretación lógica bien diferente con una y otra cópula. La relación de ligado de cuantificadores está íntimamente relacionada con el tipo de predicación (Horno Chéliz, 2011). Un PI, como (11)b, es homogéneo, como se veía antes. El hecho de que sea homogéneo impide su cuantificación (\#Los noruegos son altos tres veces a la semana) y, por lo tanto, que pueda proporcionar lecturas existenciales. (11)c, sin embargo, es una predicación télica (no homogénea), es decir, representa al sujeto de la predicación en una locación temporal. Esto permite que, al contrario que los PI como (11)b, puedan especificar un tiempo: Los noruegos están borrachos tres veces a la semana. Como señaló Husband (2012), puesto que un estadio cuantificado de un individuo es una manifestación espacio-temporal acotada del individuo, su existencia se presupone. Si se está tomando «una fracción» de la homogeneidad de un individuo, se ha de presuponer su existencia. Por ello, los PE ligan un cuantificador existencial en forma lógica.

Escandell Vidal y Leonetti (2002) excluyeron a las preposiciones de la coacción ${ }^{6}$. Estos autores, siguiendo la idea de Brenda Laca (ibidem) de que las preposiciones tienen un valor relacional, separan a estas de los estadios porque, en sus propias palabras «cuando un adjetivo relacional PI adquiere una lectura de PE, lo hace mediante la pérdida de su valor relacional, volviéndose puramente cualitativo. Dado que las preposiciones no tienen la posibilidad de ser reinterpretadas de esa forma, no pueden ser coaccionadas» (p. 170). Sin embargo, en los ejemplos de (12), se observa que sí es posible la coacción. Se debe

6. Entiéndase por coacción el proceso por el que se deriva sintácticamente un PE a partir de un PI. Como se ha visto, a partir de una predicación primaria guapo (Juan), se puede derivar, incrementando sintácticamente la estructura, [Juan [t [guapo]]] («Juan está guapo»).

LINGÜÍSTICA Y LITERATURA 
distinguir, sin embargo, (12)a, en primer lugar, como un caso excepcional en el que sí es evidente que de Madrid no puede perder su valor relacional, o lo que es lo mismo, no puede ser coaccionado sintácticamente para dejar de denotar una noción adjetival. Escandell y Leonetti (2002) se ceñían en su estudio a los casos de este tipo. Tal vez por eso excluían los SP de la lista de posibles constituyentes coaccionables.

Por otro lado, se tienen los casos (12)b, también predicaciones preposicionales que sí se prestan a la coacción. Como se observa, se puede predicar tanto un PI, como un PE añadiendo la temporalidad. (12)d y (12)e constituyen dos casos particulares. En el primer caso, lo que debe llamar la atención no es el SD mi casa, ni el hecho de que esa secuencia sea perfectamente adecuada con ser. Lo que más debe llamar la atención es por qué mi casa sí puede ser a la derecha pero mi madre no puede ser a la derecha:

(13)Mi casa es a la derecha.

(14)

a. *Mi madre es a la derecha.

b. Mi madre está a la derecha.

Evidentemente, del contraste entre (13) y (14) se desprende que el ítem léxico casa incluye en su intensión un rasgo locativo que puede ser satisfecho en la sintaxis por el sintagma a la derecha. Mi madre, sin embargo, no es una noción que de forma natural pueda adquirir en su intensión rasgos locativos — puesto que no es un individuo fijo en el espacio-, por lo que rechaza la coacción.

Por otro lado, se observa que (12)e no resulta del todo natural con estar. Si se completara de la siguiente forma: Está carta está aquí para ti, entonces resultaría más apropiada. Probablemente, se trate de un caso que incluye en su composición predicativa un rasgo preposicional (Fábregas, 2015). Entonces, aquí (la carta) y para ti (la carta), en el sentido de La carta que está aquí es para ti.

\subsection{Una propuesta formal}

A lo largo del trabajo, se ha visto cómo la diferencia esencial entre las interpretaciones de individuo y de estadio está determinada por la presencia de la variable $t$, que, como ya se 
sabe, activa el aspecto dinámico del predicado. Sin embargo, se han hecho algunas especificaciones, como la coacción sintáctica de algunos rasgos y la inclusión de nociones intensionales en la predicación sintáctica, que conviene aclarar sobre una propuesta formal.

Se empezará por los casos más evidentes: la coacción de adjetivos. Los ejemplos de (5), que se vuelven a reproducir en (15):

a. María es alegre.

b. María está alegre.

Estos dos casos se oponen por su carácter homogéneo (15)a, frente a (15)b, discontinuo o no homogéneo. Esta cuantificación de la predicación se manifiesta a través del aspecto: (15)a es atélico y (15)b es télico. Por lo tanto, la variable $t$, que se reconoce en el significado del PE, debe estar generada por un SAsp en la sintaxis.

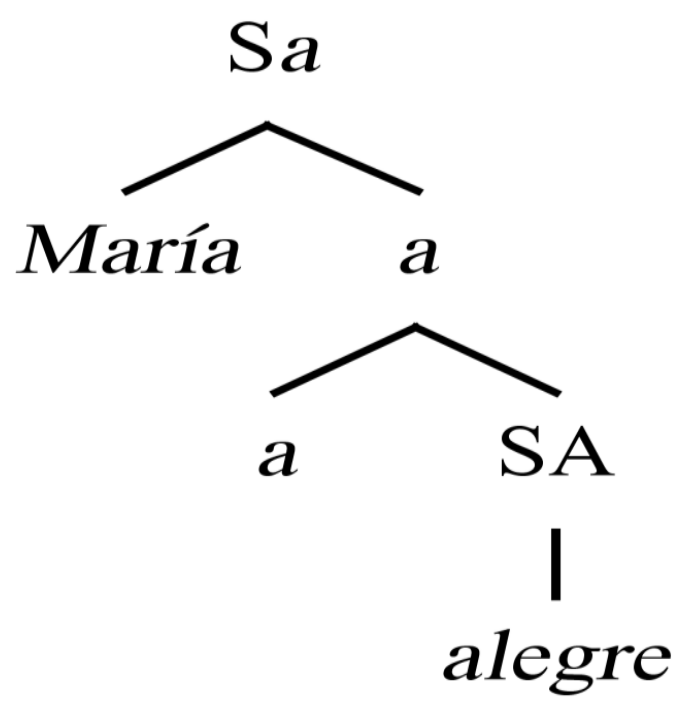

Figura 1. Diagrama correspondiente a María es alegre 


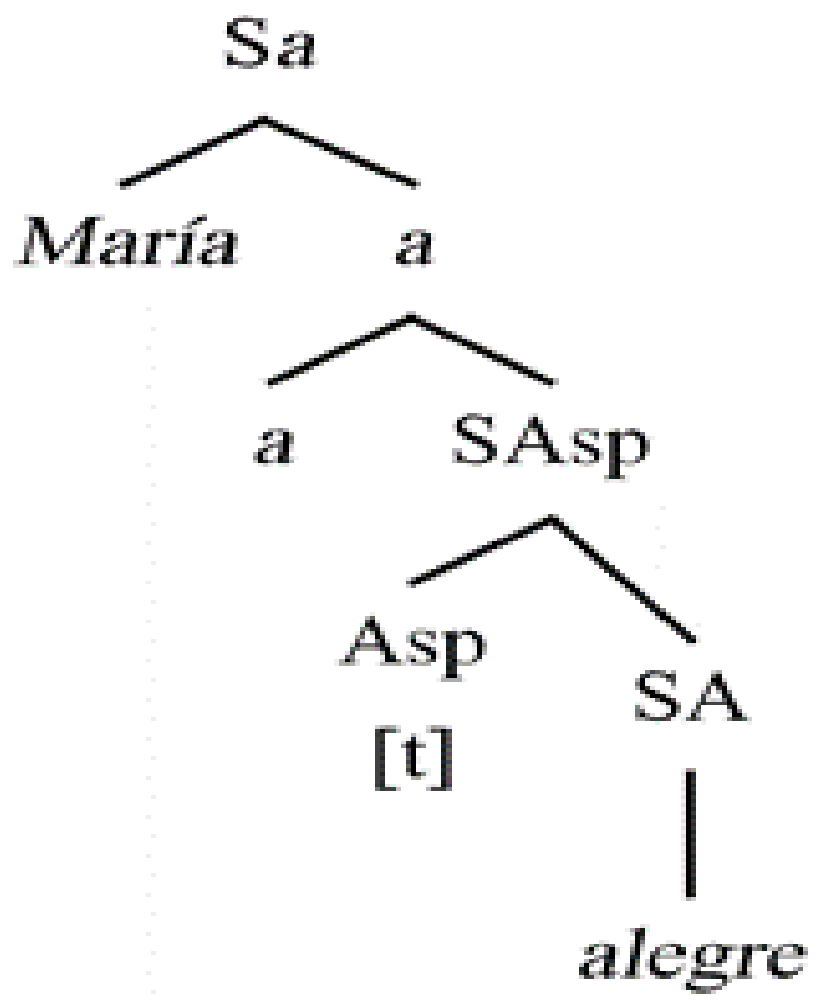

Figura 2. Diagrama correspondiente a María está alegre

En la Figura 1, se observa una predicación primaria, el SA alegre entra en combinación predicativa con el sujeto María a través de una relación de tipo adjetival ${ }^{7}$, es decir, una relación de coincidencia central. La propiedad alegre es coextensiva con María. Como se vio antes, en la relación expresada por la Figura 1, siempre que María sea María, María será feliz. Esta relación es la que se ha denominado de intensión, puesto que expresa propiedades que se reconocen como parte de los rasgos necesarios de una entidad. La Figura 1 corresponde a una lectura de individuo.

En la Figura 2, se aprecia una relación más compleja. La relación predicativa básica adjetival que se observó anteriormente no se predica de forma directa. Como se ha reafirmado a lo largo del trabajo, la relación primitiva se da entre un predicado y la variable $t$, introducida por el SAsp. Una vez que se ha ensamblado el SAsp, la predicación se

7. Para representar esa función adjetival, se utiliza el sintagma funcional Sa (con $a$ pequeña, frente al SA, de carácter léxico) propuesto por (Brucart y Hernanz, 2015, p. 80). De igual modo, para expresar una función preposicional, en el sentido de «elemento que dota a un constituyente de propiedades de la categoría preposición»se usará un $\operatorname{Sp}$ ( $p$ pequeña frente al SP léxico). 
convierte en un constituyente cuantificado, por lo que ya está listo para relacionarse con su sujeto. De esta forma, la relación ya no es coextensiva, sino que alegre se da en un tiempo $t$ y eso, a su vez, tiene como sujeto a Juan.

Estas dos estructuras, como se puede observar en los diagramas, no contienen en ningún nodo ninguna de las cópulas. Esto es así porque la cópula se especificará una vez que el predicado completo suba a SFlex para recibir los rasgos correspondientes. Como explicó Fábregas $(2012$, 2015), la función de las cópulas es precisamente dotar morfológicamente de unos rasgos temporal-aspectuales a las predicaciones mínimas que se han generado. De tal modo que, hasta donde se ha visto, para una predicación especificada con una variable $t$, la flexión generaría estar. Para una predicación sin SAsp, la flexión generaría ser. Estas mismas estructuras se aplicarían a los casos de (6), siendo que el que contenga la variable $t$ $\mathrm{y}$, por tanto, esté cuantificado gramaticalmente, podrá ligar una variable existencial en Forma Lógica y, por ende, presuponer la existencia específica de una extensión definida para el sujeto de la predicación.

Sin embargo, la coacción sintáctica no está determinada únicamente por el aspecto. Se volverá al caso (7)a, que aparecerá a continuación como (16):

\section{(16)Juan está loco.}

En este caso, la interpretación es claramente de PI: se predica una propiedad coextensiva, intensional y homogénea de un individuo. La cuestión es, entonces, por qué motivo se genera una oración con estar. La respuesta no es sencilla. En esta ocasión no se puede argumentar que es la variable $t$ la que coacciona una predicación, puesto que no existe la lectura PE esperada de esa coacción aspectual. Se debe suponer que la coacción está producida por una función preposicional. Como señaló Fábregas (2015), el verbo estar también se genera ante una coacción del tipo $p+$ PI; es decir, una predicación primaria, de la que se debería obtener una lectura de Individuo, que contiene un Sp (ver nota 7).

Fábregas (2015) explicó que el significado de (16) no es exactamente loco (Juan). Si así fuera, efectivamente, se podría aceptar Juan es loco $^{8}$. Sin embargo, la estructura de esa

8. Hay que aclarar que este estudio se está centrando en la variedad peninsular de español, donde esa secuencia es agramatical.

LINGÜÍSTICA Y LITERATURA 
oración es algo más parecido a locura (Juan); es decir, loco no es coextensivo con Juan, sino la locura es lo que coexiste en él. La propiedad intensional que se reconoce es la locura, lo que genera una estructura como la de la Figura 3. Como sucedía en los casos anteriores, una vez que esta información pasa a SFlex, la combinación de la función $\mathrm{S} p$ con los rasgos del PI generan estar en lugar de ser para expresar una predicación de Individuo.

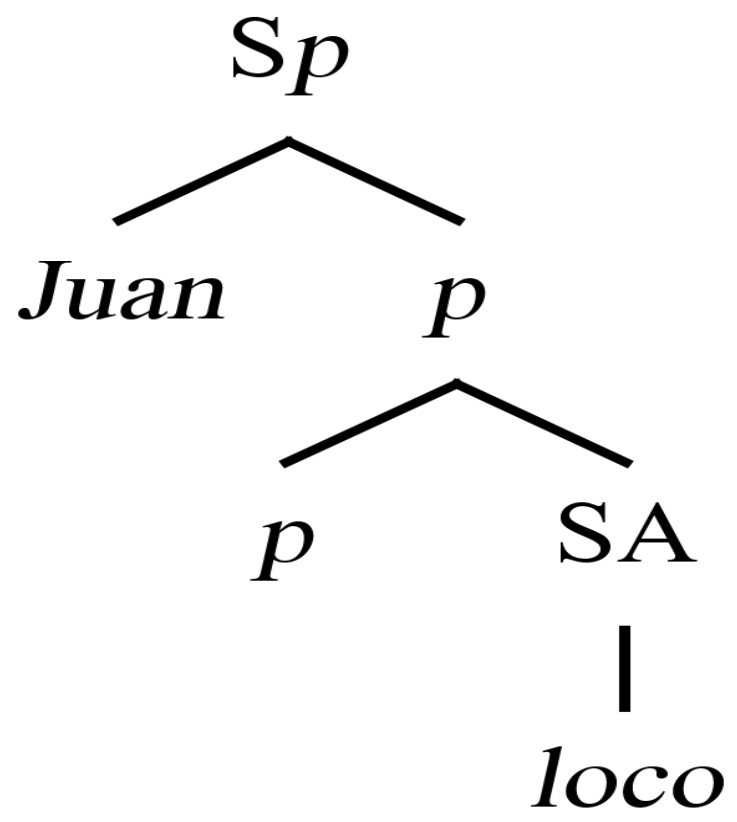

Figura 3. Diagrama correspondiente a la oración Juan está loco

Por último, se retoman los casos de (12), que aparecerán a continuación como (17):

a. María \{es/*está\} de Madrid.

b. María \{es/está\} de un inteligente.

c. María \{es/está\} de un tonto.

d. Mi casa $\{$ es/está $\}$ a la derecha.

e. Este sobre $\{$ es/?está $\}$ para ti.

Como se veía en el apartado correspondiente, los SSPP también pueden estar coaccionados por el SAsp, por lo que la propuesta de una capa aspectual que media en la predicación se puede hacer extensiva a este tipo de predicaciones. Los casos (17)a y (17)e ya se han comentado, por lo que se hará énfasis en el análisis del resto de oraciones. Dado 
que, como en los casos anteriores, se partió de un rasgo estativo no marcado - la coincidencia central y la coextensividad- (vid. las variantes con ser en (17), la capa funcional de la que se dispondrá será un $\mathrm{S} a$, que toma como elemento predicativo el SP en cuestión. Para generar la interpretación de Estadio, se le adicionará una capa aspectual intermedia.

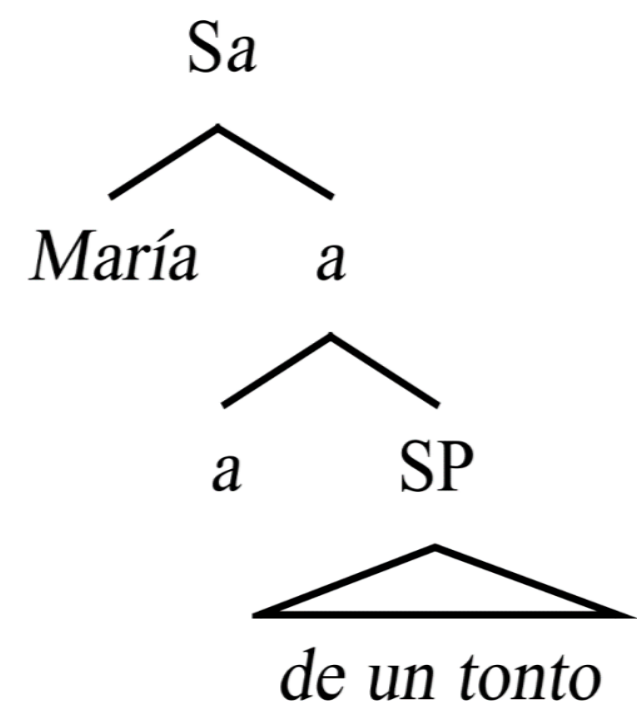

Figura 4. Diagrama correspondiente a María es de un tonto

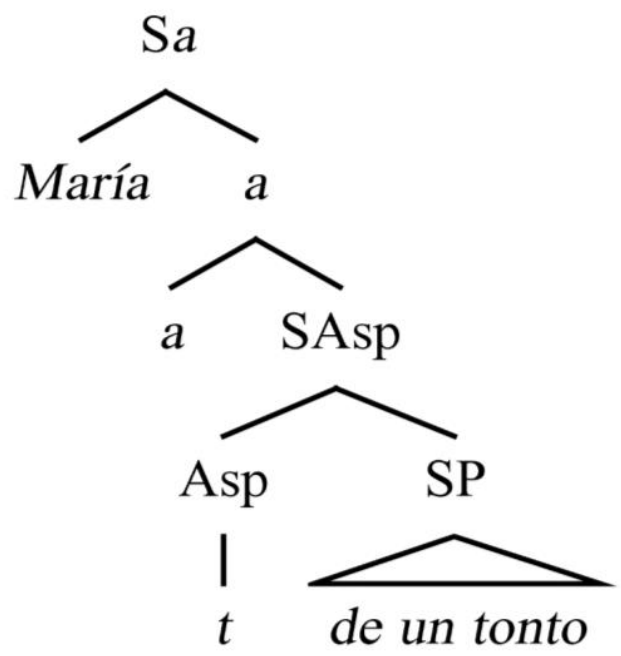

Figura 5. Diagrama correspondiente María está de un tonto 
Este esquema permite resolver los casos propuestos en (17) en sus dos lecturas: Individuo (Figura 4, léase en el sentido calificativo, no en el posesivo) y Estadio (Figura 5)

\section{Conclusiones}

Tras todo lo expuesto, se puede concluir que la predicación es un fenómeno gramatical eminentemente sintáctico basado en la operación de ensamble. Los predicados se proyectan con una serie de rasgos en la sintaxis que, durante el proceso derivacional, pueden sufrir recategorizaciones de repercusiones significativas en su interpretación semántica. El fenómeno de recategorización que se ha visto en este trabajo es el que se conoce como coacción aspectual. Si bien es habitual que en los estudios sobre el léxico se haga referencia a este proceso como coacción léxica, se evidencia que, dado que se produce en relación con las operaciones de ensamble, no es apropiado denominarlo de esa forma.

Se han revisado los principales enfoques sobre la oposición PI-PE para puntualizar que el que mejor da cuenta de una y otra forma de predicación es aquel que entra en relación con la estructura aspectual del léxico. Los PI son una forma de relación sintáctica que asigna información, y pasan a interpretarse como parte de la intensión del sujeto de la predicación o lo que es lo mismo, de coincidencia central entre propiedad y sujeto. Los PE, por su parte, incluyen un rasgo marcado de aspecto, lo que les confiere una variable espacio-temporal en la que interpretar su significado. Por ello, la propuesta que se ha expuesto defiende la existencia de una capa SAsp mediando entre el sujeto y el predicado de los PE de forma que se legitimen como eventualidades dinámicas. Existen, por otro lado, predicados que contienen el rasgo aspectual marcado en su estructura léxica, por lo que no son compatibles con un SAsp en el interior de su estructura predicativa. Esto genera dos tipos de ejemplos: por un lado, los que se derivan con ser o estar, dependiendo de si contienen una capa aspectual o no; y, por otro, los que se derivan con ser, aun cuando son aspectualmente marcados. Estos últimos coinciden con los predicados cuya estructura léxica contiene la variable $t$ o el rasgo marcado de aspecto.

Existe un caso excepcional para el que se ha tomado la explicación de Fábregas (2015). La predicación loco (Juan), en castellano, se deriva con el verbo estar, aun cuando se trata 
de una predicación que, según la argumentación que se expuso en el trabajo, es de Individuo. Se debe suponer que esta predicación contiene un rasgo prepositivo que, como se evidenció, deriva en estructuras con estar.

\section{Referencias bibliográficas}

1. Bosque, I., y Gutiérrez-Rexach, J. (2009). Fundamentos de sintaxis formal. Madrid: Akal.

2. Brucart, J. M. y Hernanz, M. L. (2015). Las posiciones sintácticas. En Á. J. Gallego (Ed.), Perspectivas de sintaxis formal (pp. 33-104). Madrid: Akal.

3. Carlson, G. (1977). Reference to Kinds in English (Tesis doctoral). University of Massachussets, Amherst.

4. Chomsky, N. (1981). Lectures on Government and Binding. Dordrecht: Foris.

5. Chomsky, N. (1995). The Minimalist Program. Cambridge (Massachusetts): The MIT Press.

6. Chomsky, N. (2001). Derivation by Phase. En M. Kenstowicz (Ed.), Ken Hale: A Life in Language (pp. 1-52). Cambridge (Massachusetts): The MIT Press.

7. Davidson, D. (1967). The Logical Form of Action Sentences. En Rescher, N. (Ed.), The Logic of Decision and Action (pp. 81-95). Pittsburgh: University of Pittsburgh Press.

8. Eguren, L. J. y Fernández Soriano, O. (2004). Introducción a una sintaxis minimista. Madrid: Gredos.

9. Escandell Vidal, M. V. \& Leonetti, M. (2002). Coercion and the Stage/Individual distinction. En J. Gutiérrez-Rexach (Ed.), From Words to Discourse (pp. 159-179). Nueva York: Elsevier.

10. Fábregas, A. (2012). A Guide to IL and SL in Spanish: Properties, Problems and Proposals. Borealis: An International Journal of Hispanic Linguistics, 1(2), 1-71.

11. Fábregas, A. (2015). On the Locative Reading of Dimensional Adjectives and the Internal Syntax of Estar. Studies in Hispanic and Lusophone Linguistics, 7(1), 3-41.

12. Frege, G. (1952). On Sense and Reference, en Translations from the Philosophical Writings of Gottlob Frege (pp. 56-78). Oxford: Blackwell. 
13. Hale, K. \& Keyser, S. J. (1987). A View from the Middle. Cambridge (Massachusetts): The MIT Presss.

14. Hale, K. \& Keyser, S. J. (1988). Explaining and Constraining the English Middle. Studies in Generative Approaches to Aspect. Cambridge (Massachusetts): The MIT Press.

15. Higginbotham, J. (1985). On Semantics. Linguistic Inquiry, 16(4), 547-593.

16. Higginbotham, J. (1989). Elucidations of Meaning. Linguistics and Philosophy, 12(4), 465-517.

17. Higginbotham, J. \& Ramchand, G. (1996). The Stage-Level/Individual-Level Distinction and the Mapping Hypothesis. Manuscrito inédito, University of Oxford.

18. Horno Chéliz, M. del C. (2011). Argumento eventivo, estados léxicos y enunciados estativo. En Á. Carrasco Gutiérrez (Ed.), Sobre estados y estatividad (pp. 63-82). München: Lincom Europa.

19. Husband, E. M. (2012). Stages of Individuals and the Composition of States. The Linguistic Review, 29(3), 375-395.

20. Kratzer, A. (1989). An Investigation of the Lumps of Thought. Linguistics and Philosophy, 12(5), 607-653.

21. Milsark, G. (1974). Existential Sentences in English (Tesis doctoral). MIT, Massachussets.

22. Parsons, T. (1985). Underlying Events in the Logical Analysis of English. In E. Lepore, \& B. P. McLaughlin (Eds.), Actions and Events: Perspectives on the Philosophy of Donald Davidson (pp. 235-267). Oxford: Blackwell.

23. Parsons, T. (1990). Events in the Semantics of English: A Study in Subatomic Semantics. Cambridge (Massachusetts): The MIT Press.

24. Pustejovsky, J. (1995). The Generative Lexicon. Cambridge (Massachusetts): MIT Press.

25. Schein, B. (1993). Plurals and Events. Cambridge (Massachusetts): MIT Press.

26. Vendler, Z. (1967). Verbs and Times. In Linguistics in Philosophy (pp. 235-267). Ithaca, NY: Cornell University Press. 